\title{
Germanica
}

GERMANICA

36 | 2005

Le pouvoir de la musique dans l'espace de langue allemande : fascination et suspicion

\section{Préface au Docteur Faustus}

Doktor Faustus nach Giacomo Manzoni, von der kritischen Untersuchung zur Inszenierung

\section{Giacomo Manzoni}

Traducteur : Laurent Feneyrou

\section{(2) OpenEdition}

\section{Journals}

Édition électronique

URL : http://journals.openedition.org/germanica/1517

DOI : 10.4000/germanica. 1517

ISSN : 2107-0784

Éditeur

Université de Lille

Édition imprimée

Date de publication : 30 juin 2005

Pagination : 101-113

ISBN : 9782913857155

ISSN : 0984-2632

Référence électronique

Giacomo Manzoni, «Préface au Docteur Faustus », Germanica [En ligne], 36 | 2005, mis en ligne le 21 mars 2014, consulté le 06 octobre 2020. URL : http://journals.openedition.org/germanica/1517 ; DOI : https://doi.org/10.4000/germanica.1517

Ce document a été généré automatiquement le 6 octobre 2020.

(c) Tous droits réservés 


\title{
Préface au Docteur Faustus
}

\author{
Doktor Faustus nach Giacomo Manzoni, von der kritischen Untersuchung zur \\ Inszenierung
}

Giacomo Manzoni

Traduction : Laurent Feneyrou

\section{NOTE DE L'AUTEUR}

\section{Note du traducteur}

Cette contribution se lit en deux temps. La première partie est un texte du compositeur Giacomo Manzoni préface au Docteur Faustus de Thomas Mann, dans une traduction de l'italien due à Laurent Feneyrou. Dans un deuxième temps, le musicologue Laurent Feneyrou propose son propre commentaire de la préface de Manzoni.

1 Sur Le Docteur Faustus est apposé, depuis sa naissance, le sceau de la catastrophe, le halo de la tragédie collective allemande et de la fin de l'art représentée par l'œuvre géniale d'Adrian Leverkühn, marqué de manière indélébile par le pacte avec le diable. Ce que confirment non seulement la littérature spécifique, à partir du fameux essai de Lukács «La tragédie de l'art moderne » en 1948, non seulement l'appréciation générale qu'en donnent les chercheurs manniens par rapport à l'évolution globale de l'écrivain, mais aussi l'autorité qui prend sa source dans la confession autobiographique et dans le sentiment personnel et intime de l'auteur. En effet, on ne compte plus, ni dans le Roman d'un roman ${ }^{1}$ ni dans les lettres de Mann, les passages où il fait explicitement allusion à l'argument sinistre et très hardi, ténébreux et étrange, développé dans le roman - par exemple, dans une lettre à Cocteau du 8 octobre 1947, « la chose la plus étrange, pour moi la plus excitante, que j'aie jamais faite ${ }^{2}$ ». Le choix d'un personnage enraciné dans la légende, le lien étroit avec l'apothéose de la barbarie nazie et avec sa débâcle apocalyptique, les personnages souvent sinistres et distants qui se pressent autour de la figure du protagoniste, sans jamais réussir à en entamer la glaciale discrétion, les thématiques choisies par le musicien pour ses compositions les plus hautes, sa vision de la «fin de l'art » qui serpente tout au long du roman et s'incarne dans la musique 
romantique qui était la plus proche du sentiment et de la formation du romancier, tout cela ne peut que plonger la biographie du compositeur allemand imaginaire dans une atmosphère sombre, presque de sabbat, qui fait honneur du reste à la tradition de la figure de Faust dans la littérature allemande notamment.

Il est difficile d'ignorer tout ce contexte critique, qui s'est peu à peu stratifié autour du roman; et il est encore plus difficile, voire aventureux, de mettre entre parenthèses les opinions de l'auteur sur son œuvre pour en proposer une autre lecture, que l'œuvre permet toutefois, étant dit que « le contenu d'une œuvre d'art commence précisément là où l'intention de l'auteur s'arrête ; elle s'éteint dans le contenu ». Un tiers de siècle après que Thomas Mann a apposé le mot «Fin » au Docteur Faustus, nous pouvons nous risquer à cette tentative et avancer de nouvelles hypothèses, sans prétendre d'ailleurs inscrire le roman dans un contexte ou lui attribuer un poids culturel et une signification spirituelle radicalement différents de ceux qui, en raison de sa nature, lui servent de compléments. Ce qui vaut surtout pour le contenu proprement historique de l'œuvre, où se réalisent concrètement les prévisions sur la fin de l'Allemagne, que Mann l'exilé - mais pas seulement lui - avait avancées bien avant qu'elles ne se vérifient. Mais le halo de tragédie, cette "apocalypsis» qui, de la composition homonyme du protagoniste, se reflète sur la réalité, ce halo vaut-il aussi pour l'art d'Adrian Leverkühn ? Pour la musique?

3 Avant de répondre à cette question, il est nécessaire de donner une image de Leverkühn, tel qu'il nous apparait, inscrit dans la réalité concrète des premières décennies de notre siècle. Ce qui ne nous intéresse pas particulièrement ici, ce sont les événements biographiques, que nous savons inspirés de Wolf, de Maupassant, et surtout de Nietzsche, même si sa naissance en 1885 pourrait faire penser à une possible relation avec Alban Berg, né à Vienne la même année.

4 Cette date n'est pas le seul fait qui pourrait conforter une telle supposition: Berg en effet, comme Leverkühn et comme d'autres personnages réels de l'histoire de la musique (parmi lesquels Schoenberg, Mahler, Puccini, Zemlinski), se rendit à Graz le 16 mai 1906 pour assister à la première autrichienne de Salomé de Strauss ; et le Concerto pour violon et orchestre attribué à Leverkühn dans le roman rappelle par certains aspects la composition du même titre de Berg. Mais l'hypothétique relation avec l'auteur de Wozzeck s'arrête ici : dans Le Journal du "Docteur Faustus", Berg est cité de manière tout à fait passagère et occasionnelle ${ }^{3}$, et il est impossible d'en déduire des implications générales en ce qui concerne la figure du protagoniste. En raison de possibles affinités avec des personnages ayant effectivement existé, il serait intéressant de plonger la figure d'Adrian Leverkühn dans l'histoire musicale du temps où Mann a voulu que sa fiction se déroule, pour en comprendre toutes les connotations et tous les développements.

5 Malgré le caractère historique et réaliste de la narration, Lerverkühn semble inscrit dans un milieu musical singulièrement privé. Certes, de nombreux personnages réels sont cités, comme les chefs d'orchestre Volkmar Andreae, Ernest Ansermet, Bruno Walter et Otto Klemperer, le mécène et chef d'orchestre Paul Sacher, le critique musical suisse Willi Schuh, et plusieurs autres; sont aussi cités les noms de musiciens ayant effectivement composé, comme Auric, de Falla, Prokofiev, Debussy ou Strauss. Mais on chercherait en vain les noms de certains grands protagonistes de la musique contemporaine, alors que la présence de Stravinski dans le roman nous incite à penser que, pour l'écrivain, les représentants authentiques et les plus avancés de la musique 
nouvelle sont précisément ceux qui ne sont pas mentionnés : Schoenberg, Berg, Webern ou Bartók (sans parler de Varèse et d'Ives, que Mann ne connaissait sans doute pas). C'est comme si Leverkühn réunissait en soi, en les absorbant, les figures qui ont le plus œuvré au renouveau radical de la musique, parmi lesquelles, en premier lieu, les trois viennois. Du reste, le roman contient des hommages explicites, nous l'avons déjà souligné, à certaines compositions de notre siècle, parmi lesquelles l'Histoire du soldat de Stravinski (dont la Gesta romanorum reprend le même ensemble instrumental), le Quatuor op. 10 de Schoenberg, pour cordes et voix soliste (à propos des deux hymnes de Keats dont il est question dans le chapitre XXVII), la Suite op. 29 du même auteur (qui utilise le même ensemble instrumental que l'Ensemblemusik de Leverkühn), ainsi que le Trio à cordes op. 45, toujours de Schoenberg, sur lequel le compositeur s'était d'ailleurs entretenu avec Thomas Mann, comme en témoigne Le Journal du "Docteur Faustus »" Pour le reste, on trouve deux hommages assez stupéfiants, et certainement « involontaires " : l'un à Schoenberg encore, dans l'Apocalypsis cum figuris, où certaines références rappellent impérieusement des pages du grand oratorio inachevé L'Échelle de Jacob, dont Mann connaissait le texte, mais pas la musique - et Adorno, qui fut le principal conseiller de Mann au moment de la rédaction du roman, ne pouvait pas connaître cette œuvre à l'époque, à moins d'avoir fouillé dans les papiers de Schoenberg, dans la maison que celui-ci habitait à Los Angeles - ; l'autre, encore plus surprenant, à Charles Ives, un compositeur dont il y a fort à parier que Mann n'avait jamais entendu la moindre note, ni vu la moindre partition, étant donné que presque toute son œuvre était alors inédite. Le second Quatuor à cordes (terminé en 1913) de ce musicien américain portait le sous-titre "Discussions»: selon les didascalies de l'auteur, nous rencontrons ici «quatre hommes qui conversent, discutent, parlent de politique, en viennent aux mains, se serrent les mains, se taisent et partent à la montagne admirer le firmament ». Or, le premier mouvement du quatuor de Leverkühn «ressemble à un dialogue très méditatif, comportant un effort intellectuel, une délibération des quatre instruments entre eux, un échange grave et paisible ${ }^{5} »:$ le parallélisme semble volontaire, même si dans ce cas, nous le disions, il ne doit s'agir que d'une coïncidence fortuite et pour le moins singulière.

6 Pour ne rien dire enfin de l'« emprunt» au dodécaphonisme schoenbergien, lequel devient pour Leverkühn un symbole technologique du renouvellement du langage musical (à partir de l'exigence de l'émancipation de la dissonance, voir le chapitre XXII), et qui donna lieu à une célèbre protestation de la part du père du dodécaphonisme, sur laquelle nous ne nous attarderons pas ici, et dont il reste un signe visible dans la note placée à la fin du roman (rappelons seulement que Mann et Schoenberg se réconcilièrent par la suite, même si ce n'est que par lettres, puisque Schoenberg mourut en juillet 1951, avant qu'il soit possible d'organiser la rencontre pour ainsi dire « publique » que les deux artistes s'étaient promis mutuellement ${ }^{6}$ ).

7 Jusque dans son évolution stylistique, Leverkühn est donc fils de son temps. L'introduction du dodécaphonisme dans le roman (celui, imaginaire, du protagoniste naît autour de 1910, l'« authentique » dix ans plus tard), mais aussi tout l'itinéraire musical de la production de Leverkühn s'enracinent solidement dans son temps historique. Il serait oiseux d'analyser les compositions engendrées dans le roman par la fantaisie de Mann et de son conseiller musical Adorno. Notons toutefois qu'il est possible d'y relever, dans le parcours stylistique du protagoniste, un parallélisme prégnant avec l'évolution du langage musical chez ses représentants les plus qualifiés : d'une œuvre d'un «épigonisme » impressionniste, « de la lignée Debussy-Ravel ${ }^{7}$ », aux 
complexes élaborations rythmico-contrapuntiques de pages ultérieures (qui peuvent évoquer le Schoenberg de la période intermédiaire, mais aussi Bartók et Hindemith), jusqu'à l'ouverture sur les mondes d'une musique nouvelle dont le dodécaphonisme n'est qu'une composante secondaire. C'est un fait que dans les descriptions des musiques de Leverkühn, les allusions à des catégories traditionnelles, comme celles de la mélodie, de la tonalité ou du rythme, diminuent au fur et à mesure au cours du roman, alors qu'émergent d'autres éléments et d'autres problématiques: dans l' Apocalypsis cum figuris et dans la Lamentatio Doctoris Fausti, les caractères du matériau apparaissent profondément modifiés. Il est évident que l'aide d'Adorno fut ici déterminante, et comme le déclare Mann dans une belle lettre du 30 décembre 1945 au jeune professeur allemand, "la notion de la musique moderne » lui fut donnée, à lui dont la «culture musicale n'a guère dépassé les années tardives du romantisme ». Dans la même lettre, Mann demande à Adorno des précisions (Exaktheiten) sur une musique avancée « supprimant la division en mesures, voire l'ordonnance des notes (glissandos des trombones); en outre, quelque chose de pratiquement presque inexécutable ; des chœurs a cappella, dans les anciens tons d'église, qui devront être chantés dans un système non tempéré en sorte que presque aucune de ces notes ou aucun de ces intervalles n'existe sur le piano ${ }^{8}$ ». En somme, Mann avait son idée, poétique, sur le nouveau matériau musical, dont Adorno se chargeait, dans les pages qu'il écrivait, de préciser les termes techniques et descriptifs, en ajoutant de son cru, et ce de manière significative. En décrivant le Concerto pour violon et orchestre, composé après l' Apocalypsis, l'analyse manienne se révèle encore rétrospective, avec son organisation harmonique pour le moins polytonale et les références à un style ancien très virtuose. Mais la vision musicale qu'il nous donne finalement de la cantate symphonique sur Faust est de nouveau très riche de trouvailles avancées et d'une conception musicale que nous dirions aujourd'hui «matiériste » et qui, par de mystérieux et de souterrains processus, semble directement se référer, par moments, aux œuvres expérimentales des générations ultérieures.

8 Dans le roman semble alors se profiler une contradiction irréductible : d'un côté, la figure éminemment tragique de Leverkühn, sur laquelle est apposée la souffrance de l'époque, le pacte avec le diable et la maladie comme nécessité et condition indispensable d'une créativité non banale (thème très ancien chez Mann), la vie du musicien se déroulant sur fond de souffrance, de luttes, et étant couronnée par une forme inexorable et dégradante de folie; de l'autre, une musique presque frétillante qui sort indemne de ces paysages d'horreur, s'élevant peu à peu vers les sommets les plus hauts, et qui parvient à donner une image vraisemblable et convaincante d'un authentique «nouveau » et d'une authentique rédemption de l'art. Les affirmations du diable ne suffisent pas pour assainir cette contradiction, affirmations selon lesquelles «il faut toujours qu'Un Tel ait été malade et dément pour que les autres n'aient plus à l'être ${ }^{9}$ ", ou que "toute une horde, toute une génération de garçons réceptifs et sains comme l'œil se précipite sur l'œuvre du génie souffrant, de l'être que la maladie a auréolé de génie, l'admire, le loue, l'exalte, l'emporte avec elle, le transforme, le lègue à la culture, laquelle ne vit pas que de pain de ménage, mais tout autant de dons et de poisons issus de la pharmacopée des "Messagers Célestes"10 ". Plus subtil, dans le livre, le fil conducteur qui mène à cette « rédemption » de la musique malgré tout le contexte tragique. Il y a en somme, ou mieux transparait en lui un élément déterminant qui modifie la manière même de considérer l'art, la musique traditionnelle, en permettant de donner une lecture avancée du pacte étroit qui lie le protagoniste avec le diable. 
Cet élément est en substance le concept de " rationalisation », d'organisation totale du matériau musical, étroitement associé dans tout le roman à une irruption de la pensée scientifique dans la musique. La clef pour la compréhension de ce processus se trouve dans la vision des choses - et pas seulement de l'art - qui est celle de Leverkühn, admirablement condensée dans l'affirmation selon laquelle "la passion la plus grande " dont est capable l'homme ne serait pas l'amour (comme le soutient le bon Zeitblom), mais la "curiosité de l'espritt"11 ${ }^{11}$. De cette position mentale naît une approche analytique, "de l'extérieur ", de la musique et de ses problèmes, avec l'œil de l'homme de science et du mathématicien plus qu'avec celui du créateur traditionnel. Chez le jeune Adrian, la «manie [...] d'imaginer des problèmes musicaux et de les résoudre comme un problème d'échecs ${ }^{12}$ » est déjà un symptôme évident de cette tendance spéculative, de celui qui se livre "à des spéculations sur la spéculatoire ${ }^{13}$ ». Son but est une modification génétique du concept d'art, selon l'analyse qu'avance Adorno dans la Philosophie de la nouvelle musique, en rapport avec l'école dont Schoenberg était le chef de file: "L'art ne veut cesser d'être une apparence et un jeu, il veut devenir une connaissance lucide ${ }^{14}$ ", au point que "l'art devient critique ${ }^{15}$ » au moment où l'œuvre pose à l'artiste « une exigence de justesse ${ }^{16}$ ». Si la musique « se rapproche beaucoup du labeur et des recherches obstinées des alchimistes et nécromants de jadis ${ }^{17}$ ", c'est là son contenu profond, auquel le compositeur du roman veut se confronter une fois pour toutes, et c'est un contenu qui devient dans ses mains simultanément un instrument décisif pour établir une conception nouvelle de la musique.

Un tel renouveau des contenus musicaux ne peut se produire qu'à travers le calcul rigoureux, l'organisation consciente du matériau, l'objectivité des procédés et la systématisation des principes opérationnels. Ces concepts reviennent dans le roman avec une fréquence impressionnante, à tel point qu'il ne semble pas nécessaire de mentionner les passages qui s'y rapportent. Mais ce qui importe, c'est de remarquer qu'à travers ces processus de rationalisation, le musicien parvient à définir précisément son concept de «composition rigoureuse": «L'intégration absolue de toutes les dimensions musicales, leur indifférence réciproque grâce à une organisation parfaite ${ }^{18}$ ». Si nous remplaçons " dimension " par " paramètre ", terme dans ce cas équivalent, il apparaît très clairement que Mann anticipe des choix techniques et compositionnels qui seront accomplis par les générations européennes gravitant, au début des " années cinquante ", autour des Cours d'été de Darmstadt. C'est la voie royale, le choix obligé qui mènera Leverkühn à la conception de son chef d'œuvre : «Le créateur du Chant de douleur du Docteur Faustus peut, dans le matériau organisé par avance, s'abandonner sans entraves, insoucieux de la structure déjà prévue, préétablie, s'abandonner à la subjectivité, et de la sorte, son œuvre la plus rigoureuse, une œuvre où le calcul est poussé à l'extrême, se trouve être en même temps purement expressive ${ }^{19}$ ", au moment même où " plus rien d'athématique ${ }^{20} »$ n'existe. Parallèlement à cette mise au point de la révolution du matériau, courent dans le livre les expressions de condamnation, sinon de rejet de la musique du passé, et surtout de la musique romantique : la description magistrale et grotesque ${ }^{21} \mathrm{du}$ prélude du troisième acte des Maîtres chanteurs de Nuremberg de Wagner n'est que le signe le plus voyant de la polémique subtile et serrée que le protagoniste mène contre la tradition romantique et d'une certaine manière contre le concept même d'expressivité, d'«inspiration", portant globalement la marque du romantisme. La musique de Leverkühn ne sait que faire de ces modèles dépassés, rhétoriques et ruisselant d'une poussive expressivité : sa voie est celle de la 
rationalité, de la lucidité critique, thèse d'où surgit une nouvelle conception non seulement du faire mais aussi du comprendre la musique.

11 Le pacte avec le diable est l'instrument essentiel pour atteindre ce but: il aiguille, précise, mais surtout glorifie les prédispositions du musicien. Ici, le diable perd ses connotations traditionnelles; son contrat ne peut plus être compris comme un contrat de perte - l'enfer comme damnation classique -, les choses sont très différentes : cet autre diable n'est que le symbole de la sécularisation de la musique, du passage de celleci au rationnel, à la conscience critique de soi. La musique sort, avec le diable du Docteur Faustus, de ses brumes irrationnelles, des cavernes de l'inconscient dont elle a toujours été l'une des principales expressions (comme elle l'a été au plus haut point de l'âme allemande, autre concept récurrent dans toute l'œuvre de Mann, suivant Schopenhauer et Nietzsche), et entre enfin dans la clarté de l'ordre, de la raison, préfigurant même la nouvelle société. Il est peut-être vrai que le cabinet du nouveau Faust « est la cuisine de sorcières dans laquelle toutes les tendances funestes de l'époque sont brassées pour en extraire leur expression la plus concentrée ${ }^{22}$ ", comme l'observait Lukács ; mais il est tout aussi vrai que par cette opération, la musique de Leverkühn atteint une autre dimension, qui n'est d'ailleurs pas un "concentré » de démonisme, mais la dimension authentique du nouveau, nécessaire et libérateur. C'est comme si «les petits», la spirochète pâle, l'infection syphilitique en somme, montant aux régions supérieures du corps, infiltrant le cerveau, les zones où la création a lieu, transfiguraient cette infection, en se sublimant et en ouvrant lentement l'« enfer » de la conscience, de la critique et d'une culture finalement libre et authentiquement laïque.

L'auteur du roman, spectateur attentif des transformations qui se produisent dans l'art, et dans la musique en particulier, se fait donc artisan récalcitrant de l'irruption du nouveau : un nouveau dont il admet la nécessité, le caractère culturel inéluctable, mais auquel il se sent personnellement étranger, incapable de le comprendre. Ainsi, cet élan déterminant vers le nouveau est consigné dans les mains du diable : c'est une tentative extrême de défense, accomplie par un homme presque trop conscient du fait que l'art a un " besoin vital de progrès révolutionnaire et de renouvellement ${ }^{23}$ ", c'est quasiment la vengeance que l'auteur des Buddenbrook, l'interprète le plus haut de la décadence bourgeoise et de la fin d'une période historique, artistique et culturelle, s'accorde par rapport à ce qui doit être désormais, malgré lui.

Profondément enraciné dans la culture traditionnelle, Thomas Mann ne pouvait pas comprendre le nouveau monde musical, esquissé par lui seul (et par Adorno), sous le signe de la positivité. Quelques mois avant sa mort, il déclarait encore à l'auteur de ces lignes, dans sa maison de Kilchberg près de Zurich, son admiration infinie pour les grands musiciens du romantisme tardif, Brahms et Bruckner en particulier, mais soutenait l'insuffisance de ses propres capacités à saisir les contenus, le sens profond des nouveaux langages. Avec son optique d'homme musicalement lié au XIX siècle, il ne pouvait pas percevoir dans les produits de son personnage, dans les thèmes chromatiques, dans les glissandi furiosi des trombones, dans les rires et dans les cris de ses chœurs, les messages d'une musique angoissée et sinistre - même si cette musique était dictée par un esprit génial -, et certainement pas le matériau régénéré qui pouvait donner vie à une nouvelle conception de la musique. Que sa perspective fut celle-ci nous est confirmé dans le livre par l'idée du « retrait », de la négation de la Neuvième Symphonie de Beethoven, à laquelle Leverkühn se prépare après la mort déchirante de son neveu Nepomuk et qui se réalise dans sa dernière cantate. Cette œuvre, qui 
représente «le bon et le noble [...], ce pour quoi les hommes ont lutté, ce pour quoi ils ont pris d'assaut les bastilles des oppresseurs, ce que les grands initiés ont annoncé en exultant ${ }^{24}$ » est annulée, niée dans la fureur créative d'une musique qui ne peut être, qui ne doit pas être noble et humaine dans le sens où le fut la Neuvième Symphonie, symbole suprême de la grande musique de la tradition à laquelle Mann se sentait lié jusqu'à la dernière goutte de son sang. Mais nous, nous pouvons affirmer aujourd'hui que la composition Lamentatio Doctoris Fausti de Leverkühn est l'œuvre qui, pour être nouvelle dans ses fibres les plus intimes, ne peut rien avoir affaire avec la Neuvième, mais doit en nier les moyens, les formes, les contenus, si elle veut effectivement poser les fondations du futur. Cette nouveauté, à laquelle Thomas Mann ne pouvait croire, il en a eu l'intuition presque contre lui-même, en laissant un message de rédemption authentique pour la musique du futur, qui est la musique d'aujourd'hui, de ceux qui, à travers les tragédies récentes de l'humanité, n'ont pas perdu la foi en ce qu'ils peuvent s'exprimer avec la voix de l'art.

Le Docteur Faustus est l'œuvre où Mann règle le plus radicalement ses comptes avec le concept d'alémanité, en vérité central dans toute sa production, avant comme après le tournant démocratique de 1922. Mais dans Le Docteur Faustus, il est sans cesse prégnant et ravage toute la conception. Non seulement parce que la musique serait une expression principalement et profondément allemande, mais parce que la guerre nazie constitue pour le romancier l'occasion définitive de révéler ce concept dans son essence. Peuple immature, introverti, obscurément attiré par des forces primitives et barbares, les Allemands ont depuis toujours constitué une présence inquiétante au cœur de l'Europe, et l'explosion de la folie nazie est d'une certaine manière la simple manifestation d'un moment caractéristique, selon Mann, constitutif de tout Allemand. Il n'y a pas selon lui de « bons » et de « mauvais » Allemands, chacun est à la fois bon et mauvais: c'est l'âme allemande qui est en soi un dangereux mélange de profond sentiment artistique et de férocité celée, de sublime capacité spéculative, philosophique et théologique, mais aussi de barbarie péniblement refoulée par bien des siècles de civilisation : «On a quelque chose en commun avec le destin allemand et la culpabilité allemande, si l'on est né allemand ${ }^{25}$ », affirme-t-il dans la conférence «L'Allemagne et les Allemands » de 1945. Le fameux discours «Frère Hitler » de 1939 n'est qu'un exemple de cette veine de la pensée manienne, laquelle le rapprochait de la position de ceux - et ils furent nombreux hors d'Allemagne - qui étaient enclins à faire porter à tout le peuple allemand les fautes des crimes commis avant et pendant le conflit par les nazis. Et ce fut la raison qui conduisit Mann à se distinguer de ces exilés démocrates qui, en 1943, proposèrent à l'opinion publique mondiale antinazie une déclaration où l'on tenait à souligner que la grande masse du peuple allemand était étrangère aux méfaits de ses tyrans (sur cet sujet, rappelons la lettre de Mann à Brecht du 10 décembre 1943 : elle creuse entre autres choses le sillon qui séparait déjà ces deux grands artistes allemands, et dont le Journal de travail de Brecht conserve des traces indélébiles et féroces ${ }^{26}$ ). Même Adrian Leverkühn, fils de la profonde Allemagne luthérienne, de ce Luther qui avait déjà, selon Mann, des "traits fortement nazis ${ }^{27}$ ", fait partie de ce destin commun et porte sur lui le poids de cette « faute collective ». De différentes manières, il rachète toutefois son peuple, ce peuple «de la contrerévolution romantique, contre l'intellectualisme philosophique et le rationalisme de l'esprit des Lumières - une révolte de la musique contre la littérature, de la mystique contre la clarté28 ", comme Mann le disait, au cours des années qui virent la naissance du roman faustien, dans la conférence "L'Allemagne et les Allemands» déjà citée. 
Leverkühn est surtout le critique le plus radical de cette alémanité ténébreuse et irrationnelle, qui est la première responsable d'une telle faute, et l'auteur met très souvent dans sa bouche des expressions de répudiation et de prise de distance qu'il n'est pas nécessaire d'énumérer ici. Il est aidé en cela par un sens très aigu de l'ironie, de la parodie, partie constitutive de toute son œuvre et de sa pensée, qui dresse un rempart insurmontable entre son moi et les possibles sursauts de ce côté le plus obscur et le plus inquiétant de l'âme allemande. Enfin, il s'isole totalement de la communauté de son peuple. Cet éloignement de la communauté, "ce repli sur soi d'Adrian, ce détachement à l'égard des problèmes de la société, cette volonté d'opposer sciemment une fin de non-recevoir à la liberté ["réalité ", selon l'édition italienne] extérieure environnante, font partie de ses traits de caractère les plus importants ${ }^{29}$ ", comme le soutient justement Lukács. Pas simplement parce que cet éloignement serait le fruit de l'atomisation du sujet dans le stade présent de la société impérialiste; mais plutôt, parce que, dirions-nous aujourd'hui, Leverkühn semble en ce sens - et peut-être inconsciemment - soit ne pas vouloir se reconnaître dans cette collectivité, soit se mettre à l'abri d'une contagion possible qui serait en vérité bien plus terrible que celle transmise par Hetaera Esmeralda. Il opère donc une sorte de distillation solitaire de son génie, qui se sépare du sort commun d'un peuple condamné à commettre des horreurs funestes et met tout en œuvre pour confectionner, presque dans les conditions aseptiques d'un laboratoire de chimiste (et non pas, malgré tout, d'alchimiste!) les conditions d'une renaissance artistique et sociale.

Adrian apparaît comme l'interprète idéal de la maxime goethéenne selon laquelle " pas de plus sûr moyen que l'art pour échapper au monde, et pas de plus sûr moyen non plus pour y être rattaché ${ }^{30} »$. Cet isolement douloureux, ce parcours tourmenté vers l'horizon de la folie (est-ce un hasard si son esprit commence à s'obscurcir en 1930, avant l'accession au pouvoir des nazis ?) est le prix à payer pour sauver la musique «d'un pompeux et solennel isolement résultant de l'émancipation de la culture, une culture promue au rang de substitut de la religion [...] s'il ne trouve un chemin pour aller vers le "peuple", c'est-à-dire, en termes moins romantiques, vers les hommes ». Cette utopie d'un art " confiant, un art à tu et à toi avec l'humanité ", Adrian l'énonce "avec un frémissement refoulé de sa voix ${ }^{31}$ ». L'humaniste Zeitblom l'accueille avec embarras, lui qui reste lié à une conception très aristocratique de l'art, conception qui reflète pleinement les convictions de Mann. Cette utopie fonde toute sa trajectoire créatrice et lui donne son sens et ses perspectives. Mais pour se réaliser, elle a besoin que naisse une autre société ; dans un discours prononcé à Berlin en 1932, résonnent ces anticipations auxquelles en 1945, au cœur même d'une catastrophe historique sans précédent dans laquelle semblent s'accomplir les plus sombres prophéties de Mann sur le destin de l'Allemagne, l'œuvre d'Adrian Leverkühn, compositeur allemand et solitaire, donne paradoxalement une force et une signification artistiques: «Le bourgeois est perdu, le monde nouveau, qui vient, qui monte, le sèmera sur sa route [...]. Le monde nouveau, le monde social, le monde organisé et planifié dans l'unité, où l'humanité sera libérée de souffrances indignes de l'homme, de peines évitables [...], sera l'œuvre de ce grand et simple bon sens réaliste que professe aujourd'hui quiconque a du poids, quiconque est choqué par les complexes sentimentaux surannés où étouffe le petit bourgeois; car pour que la vie de l'âme ait le droit de s'épanouir à nouveau et retrouve une bonne conscience dans l'évolution morale de l'homme, il faut d'abord que soit créé - ou, dans le plus mauvais cas, que s'instaure par le moyen d'une 
révolution violente - un ordre extérieur et rationnel qui soit à la hauteur des progrès accomplis par l'esprit humain ${ }^{32}$."

Mann ne va pas plus loin et ne prend pas ombrage d'une possible évolution de l'humanité vers le socialisme, mais s'aventure déjà très avant. Du reste, ce problème n'était certainement pas le sien, ni son devoir historique, même si «sa tentative d'aller au delà de lui-même et de sa propre classe, sans avoir les instruments pour le faire ${ }^{33}$ " (Asor Rosa) fut grandiose. Mais précisément, ce qui est au fond de la figure d'Adrian Leverkühn, dans ses contradictions et dans son insondable complexité d'homme et d'artiste, c'est ce message, transmis de manière idéaliste à travers les impératifs de l'art. Pour cette raison aussi, le moment est probablement venu de lire avec une autre attention, avec d'autres yeux, Le Docteur Faustus.

\section{NOTES}

1. Mann (Thomas), Le Journal du «Docteur Faustus» (1949), sous-titré «Le roman d'un roman », Paris, Christian Bourgois, 1994. Manzoni écrit ici la note suivante : « Nous renvoyons le lecteur à ce texte (qui est ici publié pour la première fois en Italie avec le Docteur Faustus sous la forme d'un seul volume), surtout en ce qui concerne les éclaircissements relatifs aux personnages qui eurent une fonction de premier plan dans la conception du roman. Cela vaut en particulier pour Theodor W. Adorno, auquel nous devons la rédaction presque littérale des descriptions de certaines compositions d'Adrian Leverkühn. Sa Philosophie de la nouvelle musique eut une influence considérable sur de nombreuses formulations de Mann, qui la lut encore dans sa version manuscrite et alors limitée à la seule partie existante (la première, intitulée "Schoenberg et le progrès"). "

2. Mann (Thomas), Lettre à Jean Cocteau du 8 octobre 1947, in Lettres 1943-1947, II, Paris, Gallimard, 1970, p. 305.

3. Mann (Thomas), Le Journal du «Docteur Faustus », op. cit., p. 70 : «Intéressé et prêt à m’apporter son concours, il [Adorno] me communique un livre "de circonstance" sur Alban Berg, qui était né la même année (1885) qu'Adrian et avec qui j'avais d'ailleurs correspondu une fois ", écrit notamment Mann.

4. Ibid., p. 206-207 : Schoenberg « me parla de son nouveau trio à peine achevé et des expériences personnelles secrètement introduites dans cette composition qui en était en quelque sorte le résultat. Il dit y avoir représenté sa maladie et les soins médicaux, y compris le male nurse l'infirmier - et tout le reste. Au demeurant, il ajouta que l'exécution serait fort ardue, voire presque impossible, ou seulement possible pour trois virtuoses consommés, mais produirait grand effet, en raison des extraordinaires sonorités. L'association des mots "inexécutable, mais à effet", je l'insérai dans le chapitre de la musique de chambre que compose Leverkühn. »

5. Mann (Thomas), Le Docteur Faustus (1947), Paris, Albin Michel, coll. « Le livre de poche », 1987, chap. XLIII, p. 598.

6. Cf. Mann (Thomas) et Schoenberg (Arnold), À propos du «Docteur Faustus», lettres 1930-1951, Lausanne, La Bibliothèque des arts, 2002.

7. Mann (Thomas), Le Docteur Faustus, op. cit., chap. XVIII, p. 211.

8. Mann (Thomas), Lettre à Theodor W. Adorno du 30 décembre 1945, in Lettres 1943-1947, op. cit., p. 211-212. Mann cite cette lettre dans Le Journal du « Docteur Faustus », op. cit., p. 146. 
9. Mann (Thomas), Le Docteur Faustus, op. cit., chap. XXV, p. 320.

10. Ibid., chap. XXV, p. 329.

11. Ibid., chap. VIII, p. 102.

12. Ibid., chap. IX, p. 108.

13. Ibid., chap. XXV, p. 335.

14. Ibid., chap. XXI, p. 250.

15. Ibid., chap. XXV, p. 325.

16. Ibid., chap. XXV, p. 325.

17. Ibid., chap. XV, p. 183.

18. Ibid., chap. XXII, p. 263.

19. Ibid., chap. XLVI, p. 638.

20. Ibid., chap. XLVI, p. 636.

21. Ibid., chap. XV, p. 186. Mann y fait allusion dans Le Journal du «Docteur Faustus », op. cit., p. 99 : "Je lus au couple Adorno et à des amis amenés par eux la correspondance entre Adrian et Kretzschmar. Le hégélien Adorno goûta la dialectique de cet échange épistolaire, mais loua plus encore l'analyse musicale qui y est incluse. Pourtant, fait étrange, il n'en reconnut point le modèle (le prélude de l'acte III des Maîtres chanteurs. "

22. Lukács (György), «La tragédie de l'art moderne » (1948), in Thomas Mann, Paris, Maspero, 1967, p. 65.

23. Mann (Thomas), Le Docteur Faustus, op. cit., chap. XV, p. 189.

24. Ibid., chap. XLV, p. 626.

25. Mann (Thomas), «L'Allemagne et les Allemands» (1945), in Les Exigences du jour, Paris, Grasset, 1976, p. 343.

26. Cf. Brecht (Bertolt), Journal de travail, Paris, L'Arche, 1976.

27. Mann (Thomas), «L'Allemagne (Germany's Guilt and Mission)» (1941, révision en 1949), in Être écrivain allemand à notre époque, Paris, Gallimard, coll. « Arcades », 1996, p. 346.

28. Mann (Thomas), «L'Allemagne et les Allemands », op. cit., p. 357.

29. Lukács (György), « La tragédie de l'art moderne », op. cit., p. 93.

30. Goethe (Wolfgang), Maximes et Réflexions («Art et littérature », 737 de la Hamburger Ausgabe des œuvres complètes de Goethe, vol. XII), Paris, Payot, 2001, p. 83.

31. Mann (Thomas), Le Docteur Faustus, op. cit., chap. XXXI, p. 428.

32. Mann (Thomas), «Goethe, représentant de l'âge bourgeois » (1932), in Noblesse de l'esprit, Paris, Albin Michel, 1960, p. 45.

33. Asor Rosa (Alberto), Thomas Mann o dell'ambiguità borghese, Bari, De Donato, 1971, p. 161.

\section{RÉSUMÉS}

Compositeur, essayiste, traducteur italien d'Adorno et de Schoenberg, Giacomo Manzoni rencontra Thomas Mann, quelques mois avant sa mort, dans sa maison de Kilchberg, et s'entretint avec lui des figures de la tradition musicale germanique, de Brahms et de Bruckner, et de sa difficulté à saisir le sens des nouveaux langages nés avec le dodécaphonisme schoenbergien. Dès lors, Le Docteur Faustus devint une source à laquelle puisèrent non seulement des textes critiques de Manzoni, portant sur les relations de Mann avec Schoenberg, ou sur sa déclinaison du mythe de Faust, et dont les enjeux culmineront en 1980 dans une riche préface à l'édition 
italienne du roman, mais aussi une œuvre scénique, Doktor Faustus, créée en 1989, à La Scala de Milan, dans une mise en scène de Robert Wilson. Cet article, en deux temps, présente la traduction française de la préface de Giacomo Manzoni à l'illustre roman, puis notre analyse du long cheminement vers l'œuvre scénique, laquelle représente le rapport entre l'artiste et sa création, cette « situation de l'art » que Mann évoquait dans une lettre à Adorno.

Der Komponist, Essayist und italienische Übersetzer Adornos und Schönbergs Giacomo Manzoni traf mit Thomas Mann wenige Monate vor dessen Tod im Haus des Schriftstellers in Kilchberg zusammen. Ihre Gespräche drehten sich um die Gestalten der deutschen Musiktradition, über Brahms und Bruckner, sowie Manns Schwierigkeiten, den Sinn der neuen Tonsprache zu verstehen, die aus Schönbergs Zwölftontechnik erwuchs. Von da an wurde der Dr. Faustus zu einer Quelle, aus der nicht nur Manzonis kritische Untersuchungen über die Beziehungen zwischen Mann und Schönberg oder über sein Verständnis des Faust-Stoffes schöpften, dessen Grundzüge 1980 in einem gehaltvollen Vorwort zur italienischen Ausgabe des Romans gipfelten, sondern auch ein Bühnenwerk: Dr. Faustus, das 1989 in einer Inszenierung von Robert Wilson an der Mailänder Scala zur Uraufführung gelangte. Der vorliegende Artikel stellt zunächst die französische Übersetzung von Manzonis Vorwort zu dem berühmten Roman vor; in einem zweiten Teil kommen wir auf unsere Analyse der langen Entstehungsgeschichte des Bühnenstücks zu sprechen, das die Be-ziehung zwischen dem Künstler und seinem Schaffen darstellt, diese « Kunstsituation », von der Thomas Mann in einem Brief an Adorno sprach. 